University of Texas at El Paso

ScholarWorks@UTEP

$9-2005$

\title{
Optimization and Decision Making under Interval and Fuzzy Uncertainty: Towards New Mathematical Foundations
}

Hung T. Nguyen

Vladik Kreinovich

The University of Texas at El Paso, vladik@utep.edu

Follow this and additional works at: https://scholarworks.utep.edu/cs_techrep

Part of the Computer Engineering Commons

Comments:

UTEP-05-02a.

Published in: Cengiz Kahraman (ed.), Fuzzy Applications in Industrial Engineering, Springer Verlag, Berlin/Heidelberg, 2006, pp. 275-290.

\section{Recommended Citation}

Nguyen, Hung T. and Kreinovich, Vladik, "Optimization and Decision Making under Interval and Fuzzy Uncertainty: Towards New Mathematical Foundations" (2005). Departmental Technical Reports (CS). 231. https://scholarworks.utep.edu/cs_techrep/231

This Article is brought to you for free and open access by the Computer Science at ScholarWorks@UTEP. It has been accepted for inclusion in Departmental Technical Reports (CS) by an authorized administrator of ScholarWorks@UTEP.For more information, please contact Iweber@utep.edu. 


\title{
Optimization and Decision Making under Interval and Fuzzy Uncertainty: Towards New Mathematical Foundations
}

\author{
Hung T. Nguyen ${ }^{1}$ and Vladik Kreinovich ${ }^{2}$ \\ 1 New Mexico State University hunguyen@nmsu.edu \\ 2 University of Texas at El Paso vladik@cs.utep.edu
}

Summary. In many industrial engineering problems, we must select a design, select parameters of a process, or, in general, make a decision. Informally, this decision must be optimal, the best for the users. In traditional operations research, we assume that we know the objective function $f(x)$ whose values describe the consequence of a decision $x$ for the user. Optimization of well-defined functions is what started calculus in the first place: once we know the objective function $f(x)$, we can use differentiation to find its maximum, e.g., as the point $x$ at which the derivative of $f$ with respect to $x$ is equal to 0 .

In real life, we often do not know the exact consequence $f(x)$ of each possible decision $x$, we only know this consequence with uncertainty. The simplest case is when we have tolerance-type (interval) uncertainty, i.e., when all we know is that the deviation between the the actual (unknown) value $f(x)$ and the approximate (known) value $f_{0}(x)$ cannot exceed the (known) bound $\Delta(x)$. In precise terms, this means that $f(x)$ belongs to the interval $\left[f_{0}(x)-\Delta(x), f_{0}(x)+\Delta(x)\right]$. In other situations, in addition to the interval that is guaranteed to contain $f(x)$, experts can also provide us with narrower intervals that contain $f(x)$ with certain degree of confidence $\alpha$; such a nested family of intervals is equivalent to a more traditional definition of fuzzy set. To solve the corresponding optimization problems, in this paper, we extend differentiation formalisms to the cases of interval and fuzzy uncertainty.

Key words: optimization, interval uncertainty, fuzzy uncertainty, extending differentiation

\section{Introduction}

Optimization and decision making are important. In many industrial engineering problems, we must select a design, select parameters of a process, or, in general, make a decision. Informally, this decision must be optimal, the best for the users. In traditional operations research, we assume that we know the objective function $f(x)$ whose values describe what is best for the users. 
For example, for a chemical plant, this function $f(x)$ may represent the profit resulting from using parameters $x$.

In such situations, the problem is to find a design (parameters, decision) $x$ that optimizes (e.g., maximizes) the given function $f(x)$ on the given range $X$.

Deterministic case: traditional approaches. Optimization of well-defined functions is what started calculus in the first place: once we know the objective function $f(x)$, we can use differentiation to find its maximum, e.g., as the point $x$ at which the derivative of $f$ with respect to $x$ is equal to 0 .

Sometimes, this equation $f^{\prime}(x)=0$ can be solved directly; sometimes, it is difficult to solve it directly, so we use gradient-based (i.e., derivatives-based) techniques to either solve this equation or to optimize the original objective function $f(x)$.

Case of probabilistic uncertainty. Often, we need to make decisions under uncertainty. In this case, we cannot predict the exact outcome $f(x)$ of a decision $x$; this outcome depends on the unknown factors. If our description of possible factors is reasonably complete, then, for each value $v$ of these unknown factors and for each decision $x$, we can predict the outcome $f(x, v)$ of the decision $x$ under the situation $v$. In the traditional approach to decision making, we assume that we can estimate the probability $p(v)$ of each situation $v$. In this case, it is reasonable to select a decision $x$ for which the expected utility $f(x) \stackrel{\text { def }}{=} \sum_{v} p(v) \cdot f(x, v)$ is the largest possible.

In many decision-making problems, instead of finitely many situations $v$, we have a continuum of possible situations - e.g., we may have one or several continuous variables like the percentage of sulfur in the oil, the oil price, the outdoors temperature, etc., that describe possible situations. In such problems, instead of finitely many probabilities $p(v)$, we have a probability distribution with a probability density $\rho(v)$, and instead of a sum, we represent the expected value as an integral $f(x)=\int_{v} \rho(v) \cdot f(x, v) d v$.

Real-life situations: beyond probabilistic uncertainty. In real life, we often do not know the probabilities of different possible situations - or at least we only have partial knowledge about these probabilities.

Interval uncertainty. The simplest case is when we have tolerance-type (interval) uncertainty, i.e., when all we know is that the deviation of actual value $x$ of the parameter (e.g., thickness of a beam) from the nominal value $x_{0}$ cannot exceed the given tolerance $\Delta$. In precise terms, this means that $x$ belongs to the interval $\left[x_{0}-\Delta, x_{0}+\Delta\right]$, an we have no information about the probabilities within this interval.

Fuzzy uncertainty. In other situations, in addition to the interval that is guaranteed to contain $x$, experts can also provide us with narrower intervals that contain $x$ with certain degree of confidence $\alpha$. Such a nested family of intervals is also called a fuzzy set, because it turns out to be equivalent to a more traditional definition of fuzzy set $[3,12,21,24,26]$ (if a traditional fuzzy set is 
given, then different intervals from the nested family can be viewed as $\alpha$-cuts corresponding to different levels of uncertainty $\alpha$ ).

Dealing with interval and fuzzy uncertainty is computationally difficult. The resulting interval and fuzzy computations techniques have been well developed, and they are still actively used in many application areas.

For foundations and applications of fuzzy techniques, see, e.g., [3, 4, 12, 26]. For applications of interval computations techniques, see, e.g., $[9,10,11,20]$.

Solving the corresponding data processing and optimization problems is often computationally difficult (NP-hard), even in the simplest case of interval uncertainty; see, e.g., [14].

At present, mostly heuristic methods are used. As a result of the abovementioned computational difficulty, to handle optimization under interval and fuzzy uncertainty, researchers mainly use heuristic techniques, techniques that often modify the techniques used in traditional optimization.

What is the problem with using heuristic techniques. Often, as a result, we get very heuristic techniques, with no clear understanding of what they are optimizing and why this particular objective function is selected to represent the original uncertain situation.

Towards new mathematical foundations. It is therefore desirable, instead of modifying techniques, to first modify the basic foundations, the main mathematical methods behind these techniques - and hopefully, justified methods will come out not just heuristic ones. In this paper, we overview the preliminary results of this research, and describe some useful mathematical techniques in detail.

Specifically:

- to optimize imprecisely defined objective functions, we must extend differential formalism to interval-valued and fuzzy-valued functions; our preliminary results are described in [15];

- to find out the utility function, we must extract a function from the data; here, we usually have linear, quadratic function, Taylor series etc. - a natural part of calculus; in uncertain case, it is often more beneficial to extract rules than expressions; see, e.g., [28];

- we must extend the expected utility theory to decision making under partially known preferences; our preliminary results are given in [7,33];

- finally, we must extend foundations of probability to the case when we only have partial information about probabilities; this topic is covered in many monographs and research papers starting with [36]; our research emphasizes computational-related aspects of these problems; see, e.g., [13, $16,17]$.

What we are planning to do. In this paper, we concentrate on extending differentiation formalisms to the interval-valued and fuzzy-valued cases.

As we have mentioned, the results of this paper were previously presented at a conference [15]. 


\section{Extending Differentiation to Interval- and Fuzzy-Valued Functions: A Problem}

In many real-life problems, we want to know the values of the derivatives. In many areas of science and engineering, we are interested in slopes. For example, a 1-D landscape is described as a dependence of the altitude $y$ on the coordinate $x$; different 1 -D landscape features are defined by different values of the slope $d y / d x$ of this dependence: low values of this slope correspond to a plain, high values to steep mountains, and medium values to a hilly terrain.

In industrial engineering, we often want to make sure that a certain parameter of a plant stays within the given range. As we monitor the value of this parameter, we would like not only to check that this value is within the range, but we also would like to look at the trend (slope) of this dependence, to be able to predict what the value of this parameter will be in the future and thus, to take preventive measures if necessary.

Interval uncertainty. In the ideal situation, when we know the exact values of $y(x)$ for every $x$, we can simply differentiate the corresponding dependence. In practice, however, the information on $y$ comes from measurements, and measurements are never exact. E.g., in the landscape example, we measure the altitudes $y_{1}, \ldots, y_{n}$ at different points $x_{1}<\ldots<x_{n}$. Since the measurements are not exact, the measured values $\widetilde{y}_{i}$ are, in general, slightly different from the the (unknown) actual altitudes $y_{i}$.

For measuring instruments, we usually have an upper bound $\Delta_{i}$ on the measurement error $\Delta y_{i} \stackrel{\text { def }}{=} \widetilde{y}_{i}-y_{i}$. This upper bound is usually provided by the manufacturer of this instrument: $\left|\Delta y_{i}\right| \leq \Delta_{i}$. Thus, after the measurement, the only information that we have about the actual (unknown) value $y_{i}$ is that this value belongs to the interval $\mathbf{y}_{i}=\left[\underline{y}_{i}, \bar{y}_{i}\right]$, where $\underline{y}_{i} \stackrel{\text { def }}{=} \widetilde{y}_{i}-\Delta_{i}$ and $\bar{y}_{i} \stackrel{\text { def }}{=} \widetilde{y}_{i}+\Delta_{i}$ (for a more detailed description of interval uncertainty, see, e.g., $[9,10,11,20])$.

Thus, the only information that we have about the actual dependence $y=f(x)$ of $y$ on $x$ is that the (unknown) function $f(x)$ belongs to the class

$$
F \stackrel{\text { def }}{=}\left\{f(x) \mid f\left(x_{i}\right) \in \mathbf{y}_{i} \text { for all } i=1, \ldots, n\right\} .
$$

We also know that the (unknown) function $f(x)$ is smooth (differentiable) because otherwise, the notion of a slope does not make sense.

In many practical applications, the derivative has a physical meaning, and this meaning implies that it is itself a continuous (or even differentiable) function. For example, when we monitor the locations $y_{i}$ of a particle at different moments of time $x_{i}$, then the derivative $d y / d x$ is a velocity; when we monitor the values $y_{i}$ of the velocity, then the derivative $d y / d x$ is the acceleration, etc. Thus, we can assume that the function $f$ is continuously differentiable.

How can we determine the slopes under such interval uncertainty? 
Toward a formal definition. Let us assume that we look for areas where the slope takes a given value $s$. In a simplified example, we monitor the location $y_{i}$ of a car on a highway at different moments of time, and we want to find out where the car was driving at the maximal allowed speed $s$ (or, alternatively, where it was driving at an excessive speed $s$ ).

Since we only know the values of the unknown function $f(x)$ at finitely many points $x_{1}<\ldots<x_{n}$, it is always possible that the derivative of the (unknown) function $f(x)$ attains the desired value $s$ at some point between $x_{i}$ and $x_{i+1}$. For example, if we are checking for the areas where the car was overspeeding, it is always possible that the car was going very fast when no one was looking (i.e., in between $x_{i}$ and $x_{i+1}$ ), for a short period of time, just for fun, so that the overall traveled distance was not affected.

In other words, for every interval $[a, b](a<b)$, it is always possible to have a function $f$ within the class $F$ (defined by the formula (1)) for which $f^{\prime}(x)=s$ for some $s \in[a, b]$.

What we are really interested in is not whether it is possible that somewhere, the slope is equal to $s$ (it is always possible), but whether the data imply that somewhere, the slope was indeed equal to $x$. This "implies" means that whatever function $f \in F$ we take, there always is a point $x \in[a, b]$ for which $f^{\prime}(x)=s$ (this point may be different for different functions $f \in F$ ).

In other words, we say that the slope is guaranteed to attain a given value $s$ somewhere on a given interval $[a, b]$ if for every function $f \in F$, the range $f^{\prime}([a, b])$ of its derivative $f^{\prime}(x)$ contains the value $s$. In mathematical terms, this means that the value $s$ belongs to the intersection of the ranges $f^{\prime}([a, b])$ corresponding to all $f \in F$.

This intersection thus describes the "range of the derivative" of the interval function $F$ on the given interval $[a, b]$. In other words, we arrive at the following definitions.

From interval to fuzzy uncertainty. As we have mentioned, interval uncertainty is just the simplest possible case of non-probabilistic uncertainty. In many real life situations, instead of an interval, we have a fuzzy set - i.e., nested family of intervals corresponding to different levels of certainty $\alpha$.

In this case, instead of an "interval function", i.e., a finite sequence of pairs $\left\langle x_{i}, \mathbf{y}_{i}\right\rangle(i=1,2, \ldots, n)$, where $x_{i}$ is a real number and $\mathbf{y}_{i}$ is an interval, we have a "fuzzy function", i.e., a finite sequence of pairs $\left\langle x_{i}, Y_{i}\right\rangle(i=1,2, \ldots, n)$, where $Y_{i}$ is a fuzzy number.

Once we define the derivative of an interval-valued function, we can naturally extend this definition to derivatives of fuzzy-valued function: Namely, for every degree of certainty $\alpha$, we consider an interval function formed by the $\alpha$-cuts of $Y_{i}$. We can then compute the "derivative" of this interval function. This derivative, as we will see, is, by itself, also an interval.

So, for each level $\alpha$, we have a derivative interval corresponding to this level $\alpha$. The nested family of these derivative intervals forms a fuzzy number - which can be thus viewed as a "derivative" of the original fuzzy function. 
In view of this comment, once we know how to define and compute derivatives of interval-valued functions, we can naturally extend this definition to fuzzy-valued functions as well. Because of this fact, in this text, we will concentrate on definitions and algorithms corresponding to interval-valued functions.

\section{Precise Formulation of the Problem}

Definition 1. By an interval function $F$, we mean a finite sequence of pairs $\left\langle x_{i}, \mathbf{y}_{i}\right\rangle(i=1,2, \ldots, n)$, where for each $i, x_{i}$ is a real number, $\mathbf{y}_{i}$ is a nondegenerate interval, and $x_{1}<x_{2}<\ldots<x_{n}$.

Definition 2. We say that a function $f: R \rightarrow R$ from reals to reals belongs to an interval function $F=\left\{\left\langle x_{1}, \mathbf{y}_{1}\right\rangle, \ldots,\left\langle x_{n}, \mathbf{y}_{n}\right\rangle\right\}$ if $f(x)$ is continuously differentiable and for every $i$ from 1 to $n$, we have $f\left(x_{i}\right) \in \mathbf{y}_{i}$.

Definition 3. Let $F$ be an interval function, and let $[a, b]$ be an interval. By $a$ derivative $F^{\prime}([a, b])$, we mean the intersection

$$
F^{\prime}([a, b]) \stackrel{\text { def }}{=} \bigcap_{f \in F} f^{\prime}([a, b])
$$

where $f^{\prime}(x)$ denotes the derivative of a differentiable function $f(x)$, and $f^{\prime}([a, b]) \stackrel{\text { def }}{=}\left\{f^{\prime}(x) \mid x \in[a, b]\right\}$ is the range of the derivative $f^{\prime}(x)$ over the interval $[a, b]$.

Comment. The notation $F^{\prime}([a, b])$ looks like the notation of a range for a realvalued function, but it is not a range: in contrast to range, if an interval is narrow enough, we can have $F^{\prime}([a, b])=\emptyset$ (see examples below).

This newly defined derivative does share some properties of the range. For example, it is well known that the range is inclusion-monotonic - in the sense that $[a, b] \subseteq[c, d]$ implies $f^{\prime}([a, b]) \subseteq f^{\prime}([c, d])$. From this property of the range, we can conclude that $[a, b] \subseteq[c, d]$ implies $F^{\prime}([a, b]) \subseteq F^{\prime}([c, d])$ - i.e., that the newly defined derivative is also inclusion-monotonic. Thus, if the union $A \cup B$ of two intervals is also an interval, we have $F^{\prime}(A \cup B) \supseteq F^{\prime}(A) \cup F^{\prime}(B)$.

Formulation of the problem. How can we compute the derivative of an interval function? The above definition, if taken literally, requires that we consider all (infinitely many) functions $f \in F$ - which is computationally excessive. Thus, we must find an efficient algorithm for computing this derivative. This is what we will do in this paper.

We will try our best to make sure that these algorithms are not simply tricks, that the ideas behind these algorithms are clear and understandable. Therefore, instead of simply presenting the final algorithm, we will, instead, present our reasoning in a series of auxiliary results that eventually leads to the asymptotically optimal algorithms for computing the desired derivative $F^{\prime}([a, b])$. 
Previous work. In our research, we were guided by results from two related research directions:

First, we were guided by different definitions of differentiation of an interval function that have been proposed by interval computations community $[2,10$, $19,22,23,27,29,30,31,32]$. The main difference from our problem is that most of these papers assume that we have intervals $\mathbf{y}$ for all $x$, while we consider a more realistic situation when the interval bounds on $f(x)$ are only known for finitely many values $x_{1}, \ldots, x_{n}$.

Second, we were guided by a paper [34] in which an algorithm was developed to check for local maxima and minima of an interval function $f$. This result has been applied to detecting geological areas $[1,5,6]$ and to financial analysis [8]. This result can be viewed as detecting the areas where the derivative is equal to $0-$ and, in this sense, as a particular case of our current problem.

\section{First Auxiliary Result: Checking Monotonicity}

Definition 4. We say that a function $f(x)$ is strongly increasing if $f^{\prime}(x)>0$ for all $x$.

Comment. Every strongly increasing function is strictly increasing, but the inverse is not necessarily true: the function $f(x)=x^{3}$ is strictly increasing but not strongly increasing.

Proposition 1. For every interval function $F$, the existence of a strongly increasing function $f \in F$ with $f^{\prime}(x)>0$ is equivalent to

$$
\underline{y}_{i}<\bar{y}_{j} \text { for all } i<j .
$$

Proof. If $f \in F$ and $f(x)$ is strongly increasing, then it is also strictly increasing hence for every $i<j$, the inequality $x_{i}<x_{j}$ implies that $f\left(x_{i}\right)<f\left(x_{j}\right)$. Since $f \in F$, we have $f\left(x_{i}\right) \in \mathbf{y}_{i}=\left[\underline{y}_{i}, \bar{y}_{i}\right]$ and $f\left(x_{j}\right) \in \mathbf{y}_{i}=\left[\underline{y}_{j}, \bar{y}_{j}\right]$. Thus, from $\underline{y}_{i} \leq f\left(x_{i}\right)<f\left(x_{j}\right) \leq \bar{y}_{j}$, we conclude that $\underline{y}_{i}<\bar{y}_{j}$, which is exactly the inequality (2).

Vice versa, let us assume that the inequalities (2) are satisfied, and let us design the corresponding strictly increasing function $f \in F$. We will first build a piece-wise linear strictly increasing function $f_{0}(x)$ for which $f_{0}\left(x_{i}\right) \in \mathbf{y}_{i}$, and then we will show how to modify $f_{0}(x)$ into a continuously differentiable strongly increasing function $f \in F$.

According to the inequalities (2), all the differences $\bar{y}_{j}-\underline{y}_{i}(i<j)$ are positive. Since all intervals are non-degenerate, the differences $\overline{\bar{y}}_{i}-\underline{y}_{i}$ are also positive. Let us denote the smallest of these positive numbers by $\Delta$. For every $i$, let us denote

$$
y_{i} \stackrel{\text { def }}{=} \max \left(\underline{y}_{1}, \ldots, \underline{y}_{i}\right)+\frac{i}{2 n} \cdot \Delta .
$$


We will then design $f_{0}(x)$ as a piece-wise linear function for which $f_{0}\left(x_{i}\right)=y_{i}$. To show that $f_{0}(x)$ is the desired piece-wise linear function, we must show that for every $i, y_{i} \in \mathbf{y}_{i}$, and that this function is strictly increasing, i.e., that $i<j$ implies $y_{i}<y_{j}$.

That $i<j$ implies $y_{i}<y_{j}$ is clear: the first (maximum) term in the formula (3) can only increase (or stay the same) when we replace $i$ by $j$, and the second term increases. Thus, it is sufficient to prove that $y_{i} \in \mathbf{y}_{i}=\left[\underline{y}_{i}, \bar{y}_{i}\right]$, i.e., that $\underline{y}_{i} \leq y_{i}$ and $y_{i} \leq \bar{y}_{i}$. We will actually prove a stronger statement: that $y_{i}<y_{i}$ and $y_{i}<\bar{y}_{i}$.

The first inequality $\underline{y}_{i}<y_{i}$ follows directly from the formula (3): by definition of a maximum, $\max \left(\underline{y}_{1}, \ldots, \underline{y}_{i}\right) \geq \underline{y}_{i}$, and when we add a positive number to this maximum, the result only increases. So, $y_{i}$ is actually larger than $\underline{y}_{i}$.

Let us now prove that $y_{i}<\bar{y}_{i}$. Indeed, by definition of $\Delta$, for all $k \leq i$, we have $\underline{y}_{k}+\Delta \leq \bar{y}_{i}$, hence (since $\left.(i / 2 n) \cdot \Delta<\Delta\right) \underline{y}_{k}+(i / 2 n) \Delta<\bar{y}_{i}$. Thus, $y_{i}-$ which is the largest of the values $\underline{y}_{k}+(i / 2 n) \Delta^{-}$is also smaller than $\bar{y}_{i}$. So, the desired $f_{0}(x)$ is designed.

Let us now show how to build the corresponding continuously differentiable function $f(x)$. For the piece-wise linear function $f_{0}(x)$, the first derivative $f_{0}^{\prime}(x)$ is piece-wise constant; since the function $f_{0}(x)$ is strictly increasing, the values $f_{0}^{\prime}(x)$ are all positive. Around each discontinuity point $x_{i}$, replace the abrupt transition with a linear one; as we integrate the resulting function, we get a new function $f(x)$ that is continuously differentiable and - since the new values of the derivative are still everywhere positive - strongly increasing. When the replacement is fast enough, the change in the value $f\left(x_{i}\right)$ is so small that $f\left(x_{i}\right)$ is still inside the desired interval $\mathbf{y}_{i}$. The proposition is proven.

Similarly, we can prove the following results:

Definition 5. We say that a function $f(x)$ is strongly decreasing if $f^{\prime}(x)<0$ for all $x$.

Proposition 2. For every interval function $F$, the existence of a strongly decreasing function $f \in F$ is equivalent to

$$
\bar{y}_{i}>\underline{y}_{j} \text { for all } i<j .
$$

Proposition 3. For every interval function $F$ and for every interval $[a, b]$, the existence of a function $f \in F$ that is strongly increasing on the interval $[a, b]$ is equivalent to

$$
\underline{y}_{i}<\bar{y}_{j} \text { for all } i<j \text { for which } x_{i}, x_{j} \in[a, b] .
$$

Proposition 4. For every interval function $F$ and for every interval $[a, b]$, the existence of a function $f \in F$ that is strongly decreasing on the interval $[a, b]$ is equivalent to

$$
\bar{y}_{i}>\underline{y}_{j} \text { for all } i<j \text { for which } x_{i}, x_{j} \in[a, b] .
$$




\section{Second Auxiliary Result: Checking Whether $0 \in \boldsymbol{F}^{\prime}([a, b])$}

Proposition 5. For every interval function $F$ and for every interval $[a, b], 0 \in$ $F^{\prime}([a, b])$ if and only if neither conditions (5) not conditions (6) are satisfied.

Proof. Let us first show that if either the conditions (5) or the conditions (6) are satisfied, then $0 \notin F^{\prime}([a, b])$.

Indeed, according to Proposition 3, if the conditions (5) are satisfied, then there exists a function $f \in F$ that is strongly increasing on $[a, b]$. For this function, $f^{\prime}(x)>0$ for all $x \in[a, b]$; therefore, $f^{\prime}([a, b]) \subseteq(0, \infty)$. Since $F^{\prime}([a, b])$ is defined as the intersection of such range sets, we have $F^{\prime}([a, b]) \subseteq$ $f^{\prime}([a, b]) \subseteq(0, \infty)$ hence $0 \notin F^{\prime}([a, b])$.

Similarly, if the conditions $(6)$ are not satisfied, then $0 \notin F^{\prime}([a, b])$.

Vice versa, let us assume that neither the conditions (5) nor the conditions (6) are satisfied, and let us show that then $0 \in F^{\prime}([a, b])$. Indeed, let $f \in F$ be an arbitrary function from the class $F$. Since the conditions (5) are not satisfied, the function $f(x)$ cannot be strongly increasing; therefore, there must be a point $x_{1} \in[a, b]$ for which $f^{\prime}\left(x_{1}\right) \leq 0$. Similarly, since the conditions $(6)$ are not satisfied, the function $f(x)$ cannot be strongly decreasing; therefore, there must be a point $x_{2} \in[a, b]$ for which $f^{\prime}\left(x_{2}\right) \geq 0$.

Since the function $f(x)$ is continuously differentiable, the continuous derivative $f^{\prime}(x)$ must attain the 0 value somewhere on the interval $\left[x_{1}, x_{2}\right] \subseteq[a, b]$. In other words, $0 \in f^{\prime}([a, b])$ for all $f \in F$. Thus, 0 belongs to intersection $F^{\prime}([a, b])$ of all possible ranges $f^{\prime}([a, b])$. The proposition is proven.

\section{Third Auxiliary Result and Final Description of $F^{\prime}([a, b])$}

Definition 6. Let $F=\left\{\left\langle x_{1}, \mathbf{y}_{1}\right\rangle, \ldots,\left\langle x_{n}, \mathbf{y}_{n}\right\rangle\right\}$ be an interval function, and let $v$ be a real number. Then, we define a new interval function $F-v \cdot x$ as follows:

$$
F-v \cdot x=\left\{\left\langle x_{1}, \mathbf{y}_{1}-v \cdot x_{1}\right\rangle, \ldots,\left\langle x_{n}, \mathbf{y}_{n}-v \cdot x_{n}\right\rangle\right\},
$$

where, for an interval $\mathbf{y}=[y, \bar{y}]$ and for a real number $c$, the difference $\mathbf{y}-c$ is defined as $[\underline{y}-c, \bar{y}-c]$.

It is easy to prove the following auxiliary result:

Proposition 6. For every interval function $F$ and for every interval $[a, b]$, $v \in F^{\prime}([a, b])$ if and only if $0 \in(F-v \cdot x)^{\prime}([a, b])$.

This results leads to the following description of the derivative $F^{\prime}([a, b])$ : 
Proposition 7. For every interval function $F$ and for every interval $[a, b]$, let $i_{0}$ and $j_{0}$ be the first and the last index of the values $x_{i}$ inside $[a, b]$. Then $F^{\prime}([a, b])=\left[\underline{F}_{i_{0}, j_{0}}, \bar{F}_{i_{0} j_{0}}\right]$, where

$$
\begin{gathered}
\underline{F}_{i_{0} j_{0}} \stackrel{\text { def }}{=} \min _{i_{0} \leq i<j \leq j_{0}} \bar{\Delta}_{i j}, \quad \bar{F}_{i_{0} j_{0}} \stackrel{\text { def }}{=} \max _{i_{0} \leq i<j \leq j_{0}} \Delta_{i j}, \\
\underline{\Delta}_{i j} \stackrel{\text { def }}{=} \frac{\underline{y}_{i}-\bar{y}_{j}}{x_{j}-x_{i}}, \quad \bar{\Delta}_{i j} \stackrel{\text { def }}{=} \frac{\bar{y}_{i}-\underline{y}_{j}}{x_{j}-x_{i}},
\end{gathered}
$$

and $[p, q] \stackrel{\text { def }}{=}\{x \mid p \leq x \& x \leq q\}$ - so when when $p>q$, the interval $[p, q]$ is the empty set.

Comment. The above expression is rather intuitively reasonable because the ratios $\underline{\Delta}_{i j}$ and $\bar{\Delta}_{i j}$ are finite differences - natural estimates for the derivatives.

Comment. As a corollary of this general result, we can conclude that if the interval $[a, b]$ contains a single point $x_{i}$ (or no points at all), then

$$
F^{\prime}([a, b])=\emptyset \text {. }
$$

Mathematically, this conclusion follows from our general result because in this case, there is no pair $i<j$, so the minimum and the maximum are taken over an empty set. By definition, the minimum of an empty set is infinite, so $\underline{F}_{i_{0} j_{0}}=+\infty$; similarly, $\bar{F}_{i_{0} j_{0}}=-\infty$. Here, $\underline{F}_{i_{0} j_{0}}>\underline{F}_{i_{0} j_{0}}$, so the interval is empty. Intuitively, however, this conclusion can be understood without invoking minima and maxima over an empty set.

Indeed, let us assume that the given interval $[a, b]$ contains only one point $x_{i}$ from the original list $x_{1}, \ldots, x_{n}$. Then, for any real number $s$, we can take, as $f \in F$, a function that takes an arbitrary value $y_{i} \in \mathbf{y}_{i}$ for $x=x_{i}$ and that is linear with a slope $s$ on $[a, b]$ - i.e., the function

$$
f(x)=y_{i}+s \cdot\left(x-x_{i}\right) .
$$

For this function $f(x)$, the range $f^{\prime}([a, b])$ of the derivative $f^{\prime}(x)$ on the interval $[a, b]$ consists of a single point $s$. Thus, if we take two such functions corresponding to two different values of $s$, then the intersection of their ranges is empty. Therefore, the range $F^{\prime}([a, b])$ - which is defined (in Definition 3) as the intersection of all such ranges $f^{\prime}([a, b])$ - is also empty.

Proof. The fact that conditions (5) are not satisfied means that there exist value $i_{0} \leq i<j \leq j_{0}$ for which $\underline{y}_{i} \geq \bar{y}_{j}$. The fact that the conditions (6) are not satisfied means that there exist values $i_{0} \leq i^{\prime}<j^{\prime} \leq j_{0}$ for which $\bar{y}_{i^{\prime}} \leq \underline{y}_{j^{\prime}}$.

Similarly, the fact that the conditions (5) and (6) are not satisfied for the interval function $F-v \cdot x$ mean that

$$
\exists i, j\left(i_{0} \leq i<j \leq j_{0} \& \underline{y}_{i}-v \cdot x_{i} \geq \bar{y}_{j}-v \cdot x_{j}\right)
$$


and

$$
\exists i^{\prime}, j^{\prime}\left(i_{0} \leq i^{\prime}<j^{\prime} \leq j_{0} \& \bar{y}_{i^{\prime}}-v \cdot x_{i^{\prime}} \leq \underline{y}_{j^{\prime}}-v \cdot x_{j^{\prime}}\right) .
$$

The inequality $\underline{y}_{i}-v \cdot x_{i} \geq \bar{y}_{j}-v \cdot x_{j}$ can be described in the equivalent form $v \cdot\left(x_{j}-x_{i}\right) \geq \bar{y}_{j}-\underline{y}_{i}$, i.e., since $x_{i}<x_{j}$, in the form $v \geq \bar{\Delta}_{i j}$. Thus, the existence of $i$ and $j$ as expressed by the formula (9) can be described as the existence of $i$ and $j$ for which $v$ is larger than the corresponding value $\bar{\Delta}_{i j}$, i.e., as

$$
v \geq \min _{i_{0} \leq i<j \leq j_{0}} \bar{\Delta}_{i j}
$$

Similarly, the condition (10) is equivalent to

$$
v \leq \max _{i_{0} \leq i<j \leq j_{0}} \underline{\Delta}_{i j}
$$

The proposition is proven.

\section{Towards a Faster Algorithm}

Proposition 7 provides an explicit formula for computing $F^{\prime}([a, b])$ for each interval $[a, b]$. For each $[a, b]$, we need to compute $O\left(n^{2}\right)$ values of $\Delta_{i j}$ and $\bar{\Delta}_{i j}$.

In problem like locating landscape features, we are not so much interested in knowing whether a given type of landscape exists in a given zone, but rather in locating all types of landscape. In other words, we would like to be able to find the values $F^{\prime}([a, b])$ for all possible intervals $[a, b]$. According to Proposition 7 , it is sufficient to find all the values $F^{\prime}\left(\left[x_{i_{0}}, x_{j_{0}}\right]\right)$ for all $i_{0}, j_{0}=1, \ldots, n$ for which $i_{0}<j_{0}$. There are $n \cdot(n+1) / 2=O\left(n^{2}\right)$ such values. If we use the formula from Proposition 7 - that takes $O\left(n^{2}\right)$ computational steps - to compute each of these $O\left(n^{2}\right)$ values, we will need an overall of $O\left(n^{2}\right) \cdot O\left(n^{2}\right)=O\left(n^{4}\right)$ steps.

For large $n$-e.g., for $n \approx 10^{6}$ - we need $n^{4} \approx 10^{24}$ computational steps; this is too long for even the fastest computers. Let us show that we can compute the interval derivative faster, actually in $O\left(n^{2}\right)$ time. Since we must return $O\left(n^{2} 0\right.$ results, we cannot do it in less than $O\left(n^{2}\right)$ computational steps - so this algorithm is (asymptotically) optimal.

Proposition 8. There exists an algorithm that, given an interval function $F=\left\{\left\langle x_{1}, \mathbf{y}_{1}\right\rangle, \ldots,\left\langle x_{n}, \mathbf{y}_{n}\right\rangle\right\}$, computes all possible values of the derivative $F^{\prime}([a, b])$ in $O\left(n^{2}\right)$ computational steps.

Proof. At first, we compute $O\left(n^{2}\right)$ values $\Delta_{i j}$ and $\bar{\Delta}_{i j}$ by using the formulas (8); this requires $O\left(n^{2}\right)$ steps.

Let us now show how to compute all $n^{2}$ values $\bar{F}_{i_{0} j_{0}}$ in $O\left(n^{2}\right)$ steps.

First, for each $i$, we sequentially compute the "vertical" maxima $\bar{v}_{i j} \stackrel{\text { def }}{=}$ $\max \left(\underline{\Delta}_{i, i+1}, \ldots, \underline{\Delta}_{i j}\right)$ corresponding to $j=i+1, i+2, \ldots, n$ as follows: $\bar{v}_{i, i+1}=$ 
$\underline{\Delta}_{i, i+1}$ and $\bar{v}_{i j}=\max \left(\bar{v}_{i, j-1}, \underline{\Delta}_{i j}\right)$ for $j>i+1$. For each $i=1, \ldots, n$, to compute all these values, we need $\leq n$ computational steps. Thus, to compute all such values $\bar{v}_{i j}$ for all $i$ and $j$, we need $\leq n \cdot n=O\left(n^{2}\right)$ computational steps.

Then, for every $j_{0}$, we sequentially compute the values $\bar{F}_{i_{0} j_{0}}$ for $i_{0}=j_{0}-$ $1, j_{0}-2, \ldots, 1$ as follows: $\bar{F}_{j_{0}-1, j_{0}}=\underline{v}_{j_{0}-1, j_{0}}$ and $\bar{F}_{i_{0}, j_{0}}=\max \left(\bar{F}_{i_{0}+1, j_{0}}, \bar{v}_{i_{0}, j_{0}}\right)$ (it is easy to see that this formula is indeed correct). For each $j_{0}=1, \ldots, n$, to compute all these values, we need $\leq n$ computational steps. Thus, to compute all such values $\bar{F}_{i j}$ for all $i_{0}$ and $\bar{j}_{0}$, we need $\leq n \cdot n=O\left(n^{2}\right)$ computational steps.

Similarly, by using $\bar{\Delta}_{i j}$ instead of $\Delta_{i j}$ and min instead of max, we can compute all $n^{2}$ values $\underline{F}_{i_{0} j_{0}}$ in $O\left(n^{2}\right)$ steps. The proposition is proven.

\section{This Same Differential Formalism Also Serves an Alternative Definition of Zones}

In some practical problems, a zone is defined not by an exact value of the derivative $v$, but an interval $\mathbf{v}=[\underline{v}, \bar{v}]$ of possible values. In this case, it makes sense to say that an interval $[a, b]$ contains a zone if for every function $f \in F$, there is at least one point $x \in[a, b]$ for which $f^{\prime}(x) \in \mathbf{v}$. In other words, we say that the interval $[a, b]$ contains a zone of a given type if $f^{\prime}([a, b]) \cap \mathbf{v} \neq \emptyset$ for all functions $f \in F$.

It turns out that the above notion of a derivative can help us detect such zones as well. Namely, the following statement is true:

Proposition 9. For every interval function $F$ and for every two intervals $[a, b]$ and $\mathbf{v}$, the following properties are equivalent to each other:

- for every function $f \in F$, we have $f^{\prime}([a, b]) \cap \mathbf{v} \neq \emptyset$;

- $\underline{F}_{i_{0} j_{0}} \leq \bar{v}$ and $\bar{F}_{i_{0} j_{0}} \geq \underline{v}$.

Proof. We will prove the equivalence of the two opposite statements:

- there exists a function $f \in F$ for which $f^{\prime}([a, b]) \cap \mathbf{v}=\emptyset$;

- $\underline{F}_{i_{0} j_{0}}>\bar{v}$ or $\bar{F}_{i_{0} j_{0}}<\underline{v}$.

Indeed, let us assume that there exists a function $f \in F$ for which $f^{\prime}([a, b]) \cap$ $\mathbf{v}=\emptyset$. Since every function $f \in F$ is continuously differentiable, its derivative $f^{\prime}(x)$ is a continuous function, hence the range $f^{\prime}([a, b])$ is an interval. There are two possible situations when this interval range does not intersect with $\mathbf{v}$ :

- either all the values from this range are $>\bar{v}$,

- or all the values from this range are $<\underline{v}$.

In the first case, we have $f^{\prime}(x)>\bar{v}$ for all $x \in[a, b]$. Therefore, for the function $g(x) \stackrel{\text { def }}{=} f(x)-\bar{v} \cdot x$, we get $g^{\prime}(x)>0$ for all $x$, i.e., the function 
$g(x)$ is strongly increasing. Since $f \in F$, we have $g \in G \stackrel{\text { def }}{=} F-\bar{v} \cdot x$. Due to Proposition 1, the existence of a strongly increasing function $g \in G$ means that $\underline{y}_{i}-\bar{v} \cdot x_{i}<\bar{y}_{j}-\bar{v} \cdot x_{j}$ for all $i<j$. This inequality, in its turn, means that $\bar{\Delta}_{i j}>\bar{v}$ for all $i<j$. Thus, $\bar{v}$ is smaller than the smallest of the values $\bar{\Delta}_{i j}$, i.e., smaller than $\underline{F}_{i_{0} j_{0}}$. $\bar{F}_{i_{0} j_{0}}<\underline{v}$.

Similarly, in the second case, we have $f^{\prime}(x)<\underline{v}$ for all $x \in[a, b]$, hence

Vice versa, let $\underline{F}_{i_{0} j_{0}}>\bar{v}$. By definition of $\underline{F}_{i_{0} j_{0}}$ as the minimum, this means that $\bar{\Delta}_{i j}>\bar{v}$ for all $i, j$ for which $i_{0} \leq i<j \leq j_{0}$. Substituting the definition of $\bar{\Delta}_{i j}$, multiplying both sides of the inequality by a positive term $x_{j}-x_{i}$ and moving terms to another side, we conclude that $\underline{y}_{i}-\bar{v} \cdot x_{i}<\bar{y}_{j}-\bar{v} \cdot x_{j}$ for all $i<j$. This inequality, in its turn, means that for the interval function $G \stackrel{\text { def }}{=} F-\bar{v} \cdot x$, formula (2) holds and thus, due to Proposition 1, there exist a strongly monotonic function $g \in G$ for which $g^{\prime}(x)>0$ for all $x$. Then, for the function $f(x) \stackrel{\text { def }}{=} g(x)+\bar{v} \cdot x$, we have $f \in F$ and $f^{\prime}(x)=g^{\prime}(x)+\bar{v}>\bar{v}$ for all $x$ - hence, $f^{\prime}([a, b]) \cap \mathbf{v}=\emptyset$.

Similarly, if $\bar{F}_{i_{0} j_{0}}<\underline{v}$, there exists a function $f \in F$ for which $f^{\prime}([a, b]) \cap$ $\mathbf{v}=\emptyset$. The proposition is proven.

\section{Open Problems}

What if we take into consideration uncertainty in measuring $x$ ? In the above text, we took into consideration the uncertainty of measuring $y$, but assumed that we know $x$ exactly. In real life, there is also some uncertainty in measuring $x$ as well. How can we take this uncertainty into consideration?

For the problem of finding local minima and maxima, this uncertainty was taken into consideration in [18]. It is desirable to extend this approach to finding the range of the derivatives.

Parallelization. In the above text, we described how to compute the derivative of an interval function in time $O\left(n^{2}\right)$, where $n$ is the number of observations, and showed that this algorithm is (asymptotically) optimal in the sense that no algorithm can compute this derivative faster.

For reasonable $n$, e.g., for $n \approx 10^{3}, n^{2}$ computational steps means a million steps; it is quite doable on modern computers. However, for large $n$, e.g., for $n \approx 10^{6}, n^{2}$ computational steps is $10^{12}$ steps, so on a modern Gigaherz machine, the corresponding computations will take $10^{3} \mathrm{sec}$ - almost an hour.

How can we further speed up the corresponding computations? Our optimality result shows that we cannot achieve a drastic speed-up if we use sequential computers. Thus, the only way to speed up the corresponding computations is to use parallel computers.

For the problem of finding local minima and local maxima, parallel computers can indeed speed up the corresponding computations; see, e.g., [35]. 
An important question is therefore: How can speed up the computation of the corresponding derivative by using parallel computers?

\section{Acknowledgments}

This work was supported in part by NASA under cooperative agreement NCC5-209, by NSF grants EAR-0112968, EAR-0225670, and EIA-0321328, and by NIH grant 3T34GM008048-20S1. The authors are also thankful to participants of the interval computations mailing list, especially to R. Baker Kearfott, Svetoslav Markov, Arnold Neumaier, and Andrei Sobolevskii, for valuable comments.

\section{References}

1. Aguiar MS, Costa ACR, Dimuro GP (2004) ICTM: an interval tessellation-based model for reliable topographic segmentation. Numerical Algorithms 37:3-11

2. Angelov R, Markov S (1981) Extended Segment Analysis. Freiburger IntervallBerichte 81(10):1-63.

3. Bojadziev G, Bojadziev M (1995). Fuzzy sets, fuzzy logic, applications, World Scientific, Singapore

4. Booker J, Parkinson J, Ross TJ, eds. (2002) Combined fuzzy logic and probability applications. SIAM, Philadelphia

5. Coblentz DD, Kreinovich V, Penn BS, Starks SA (2000) Towards reliable subdivision of geological areas: interval approach. In: Proc. NAFIPS'2000, Atlanta, Georgia, July 13-15, 2000, 368-372.

6. Coblentz DD, Kreinovich V, Penn BS, Starks SA (2003) Towards reliable subdivision of geological areas: interval approach, In: Reznik L, Kreinovich V (eds.), Soft Computing in Measurements and Information Acquisition, Springer-Verlag, Berlin-Heidelberg, 223-233.

7. de la Mora C, Wojciechowski P, Kreinovich V, Starks SA, Tanenbaum P, Kuzminykh A (2003) Robust methodology for characterizing system response to damage: a subjective (fuzzy) partial ordered modification of the traditional utility-probability scheme. In: Proceedings of the 22nd International Conference of the North American Fuzzy Information Processing Society NAFIPS'2003, Chicago, Illinois, July 24-26, 2003, 413-419.

8. Deboeck GJ, Villaverde K, Kreinovich K (1995) Interval methods for presenting performance of financial trading systems. Reliable Computing, Supplement (Extended Abstracts of APIC'95: International Workshop on Applications of Interval Computations, El Paso, TX, Febr. 23-25, 1995), 67-70.

9. Jaulin L, Kieffer M, Didrit O, Walter E (2001) Applied interval analysis: with examples in parameter and state estimation, robust control and robotics. Springer, London

10. Kearfott RB (1996) Rigorous global search: continuous problems. Kluwer, Dordrecht

11. Kearfott Rb, Kreinovich V., eds (1996) Applications of interval computations. Kluwer, Dordrecht 
12. Klir G, Yuan, B (1995) Fuzzy sets and fuzzy logic: theory and applications. Prentice Hall, Upper Saddle River, New Jersey

13. Kreinovich V (2004) Probabilities, intervals, what next? optimization problems related to extension of interval computations to situations with partial information about probabilities. Journal of Global Optimization 29(3):265-280

14. Kreinovich V, Lakeyev A, Rohn J, Kahl P (1997) Computational complexity and feasibility of data processing and interval computations. Kluwer, Dordrecht

15. Kreinovich V, Nguyen HT, Dimuro GP, da Rocha Costa AC, Bedregal BRC (2003) A new differential formalism for interval-valued functions and its potential use in detecting 1-D landscape features. In: Proceedings of the International Conference on Information Technology InTech'03, Chiang Mai, Thailand, December 17-19, 2003, 491-498.

16. Kreinovich V, Nguyen HT, Ferson S, Ginzburg L (2003) From computation with guaranteed intervals to computation with confidence intervals: a new application of fuzzy techniques. In: Proceedings of the 21st International Conference of the North American Fuzzy Information Processing Society NAFIPS'2002, New Orleans, Louisiana, June 27-29, 2002, 418-422

17. Kreinovich V, Nguyen HT, Wu B (to appear) On-line algorithms for computing mean and variance of interval data, and their use in intelligent systems, Information Sciences

18. Lorkowski J, Kreinovich V (1996) If we measure a number, we get an interval. What if we measure a function or an operator? Reliable Computing 2(3):287-298

19. Markov S (1979) Calculus for interval functions of a real variable. Computing 22:325-337.

20. Moore RE (1979) Methods and applications of interval analysis. SIAM, Philadelphia

21. Moore RE, Lodwick WA (2003) Interval analysis and fuzzy set theory. Fuzzy Sets and Systems 135(1):5-9.

22. Muñoz H, Kearfott RB (2004) Slope intervals, generalized gradients, semigradients, and csets. Reliable Computing 10(3):163-193

23. Neumaier A (2001) Introduction to numerical analysis. Cambridge Univ. Press, Cambridge

24. Nguyen HT, Kreinovich V (1996) Nested intervals and sets: concepts, relations to fuzzy sets, and applications, In: Kearfott, RB, Kreinovich, V (eds.), Applications of interval computations. Kluwer, Dordrecht, 245-290

25. Nguyen HT, Kreinovich V (1999) How to divide a territory? A new simple differential formalism for optimization of set functions. International Journal of Intelligent Systems 14(3):223-251.

26. Nguyen HT, Walker, EA (1999) First course in fuzzy logic, CRC Press, Boca Raton, Florida

27. Ratz D (1998) Automatic slope computation and its application in nonsmooth global optimization, Shaker-Verlag, Aachen

28. Salvatore AP, Biswas A, Kreinovich V, Manriquez B, Cannito MP, Sinard RJ (2004) Expert system-type approach to voice disorders: scheduling botulinum toxin treatment for adductor spasmodic dysphonia. In: Proceedings of the Fifth International Conference on Intelligent Technologies InTech'04, Houston, Texas, December 2-4, 2004.

29. Sendov B (1977) Segment arithmetic and segment limit. C. R. Acad. Bulg. Sci. 30:955-968. 
30. Sendov B (1977) Segment derivatives and Taylor's formula. C. R. Acad. Bulg. Sci. 30:1093-1096.

31. Sendov B (1980) Some topics of segment analysis. In: Nickel K (ed.), Interval mathematics'80, Academic Press, N.Y., 236-245.

32. Sendov B (1990) Hausdorff approximations. Kluwer, Dordrecht

33. Tanenbaum PJ, de la Mora C, Wojciechowski P, Kosheleva O, Kreinovich V, Starks SA, Kuzminykh A (2004) Robust methodology for characterizing system response to damage: approach based on partial order. In: Lirkov I, Margenov S, Wasniewski J, Yalamov P (eds.), Large-Scale Scientific Computing, Proceedings of the 4-th International Conference LSSC'2003, Sozopol, Bulgaria, June 4-8, 2003, Springer Lecture Notes in Computer Science 2907:276-283.

34. Villaverde K, Kreinovich V (1993) A linear-time algorithm that locates local extrema of a function of one variable from interval measurement results. Interval Computations, No. 4:176-194.

35. Villaverde K, Kreinovich V (1995) Parallel algorithm that locates local extrema of a function of one variable from interval measurement results. Reliable Computing, Supplement (Extended Abstracts of APIC'95: International Workshop on Applications of Interval Computations, El Paso, TX, Febr. 23-25, 1995), 212-219.

36. Walley P (1991) Statistical reasoning with imprecise probabilities, Chapman \& Hall, New York 